\title{
M-COMMERCE: UMA REVISÃO DA LITERATURA FOCADA NOS OFERTANTES \\ DO SERVIÇO
}

\author{
M-COMMERCE: A LITERATURE REVIEW FOCUSED ON THE SERVICE \\ OFFERORS
}

\section{M-COMMERCE: UNA REVISIÓN BIBLIOGRÁFICA CENTRADA EN LOS} OFRECENTES DEL SERVICIO

\section{Wilnei Aldir Schneider}

Doutorando em Administração pela

Universidade do Estado de Santa Catarina

(UDESC), Brasil;

was.was@hotmail.com

\section{Rafael Tezza}

Doutor em Engenharia da Produção pela

Universidade Federal de Santa Catarina

(UFSC), Brasil; Professor Adjunto da UDESC

rafael.tezza@udesc.br
Contextus

ISSNe 2178-9258

Organização: Comitê Científico Interinstitucional Editor Científico: Carlos Adriano Santos Gomes Avaliação : Double Blind Review pelo SEER/OJS Revisão: Gramatical, normativa e de formatação Recebido em 02/06/2016 Aceito em 19/09/2016 $2^{\text {a }}$ versão aceita em 09/12/2016

\section{RESUMO}

Esta pesquisa teve o objetivo de identificar vantagens e desafios que as organizações encontram ao oferecerem o serviço de m-commerce. Foi realizada busca sistemática na literatura e bibliometria, classificando os 16 trabalhos encontrados, e análise descritiva de conteúdo, apresentando e discutindo os resultados reportados nestes artigos. Quanto aos resultados deste estudo, podem ser elencados os seguintes: há poucos estudos que analisam os ofertantes do serviço de m-commerce; não existe uma revista específica que publica estudos com o foco aqui tratado; as pesquisas não são focadas em um setor empresarial específico; há diversos benefícios que uma empresa pode obter ao oferecer o serviço de $\mathrm{m}$-commerce, entretanto, também há barreiras que necessitam ser superadas; e algumas diretrizes devem ser seguidas para que se obtenha sucesso no mercado de m-commerce.

Palavras-chave: $M$-commerce. Revisão de literatura. Serviço móvel. Administração. Comércio eletrônico.

\begin{abstract}
This research aimed at the identification of advantages and challenges that organizations face when offering m-commerce service. We conducted a systematic search in the literature and a bibliometric study. The sixteen articles found were classified and analyzed descriptively by content analysis, so that the reported results could be presented and discussed. Regarding the results of this research, the following ones may be listed: there are few studies that analyze the providers of $\mathrm{m}$-commerce service; there is no specific journal that publishes studies with this specific focus; the existing researches are not focused on a specific business sector; there are several benefits that a company may obtain by offering m-commerce, however, there are also barriers that need to be overcome; and some guidelines might be followed for the success of the m-commerce market.
\end{abstract}

Keywords: M-commerce. Literature review. Mobile service. Administration. Electronic commerce. 


\section{RESUMEN}

Esta investigación tuvo como objetivo identificar las ventajas y desafíos que encuentran las organizaciones que ofrecen un servicio de $m$-commerce. Fue realizada una búsqueda sistemática en la literatura, bibliometría, la clasificación de las 16 obras que se encuentran, y análisis de contenido descriptivo, con presentación y discusión de los resultados presentados en estos artículos. Cuanto a los resultados de este estudio, se puede enumerar los siguientes: hay pocos estudios que analizan los ofrecentes de servicios de $\mathrm{m}$-commerce; no existe ninguna revista específica que publica estudios con el foco del presente estúdio; las investigaciones no se centran en un sector de actividad específica; hay varios beneficios que una empresa puede obtener con la prestación del servicio de m-commerce, sin embargo, también hay barreras que hay que superar; y algunas pautas deben seguirse para obtener el éxito en el mercado de mcommerce.

Palabras clave: M-commerce. Revisión de literatura. Servicio móvil. Administración. Comercio electrónico.

\section{INTRODUÇÃO}

A partir de 1990, o comércio, da forma como era conhecido até então, passou por uma grande mudança com a chegada do comércio eletrônico (e-commerce), que trouxe uma nova forma de conduzir os negócios (NGAI; GUNASEKARAN, 2005). Com o tempo, novas tecnologias foram surgindo, dentre elas, os dispositivos móveis com acesso sem fio à internet (FROLICK; CHEN, 2004). Essas tecnologias, além de marcar uma era de comunicação em massa, também têm marcado uma era de comércio para as massas, pois o que antes exigia um ponto fixo de conexão à internet, com os dispositivos móveis (celulares, smartphones, tablets e outros que permitam o acesso móvel à internet) pode ser feito a partir de qualquer lugar (BHEDA, 2010).

Com isso, surgiu uma nova forma de comércio, o m-commerce (Mobile
Commerce), que é uma forma específica de comércio eletrônico realizado por meio de dispositivos móveis que tenham conexão com a internet (CHONG, 2013; IVAN; MILODIN; ZAMFIROIU, 2013; JONKER, 2003; MAY, 2001). Isso fez surgir uma variedade de estudos, dentre os quais alguns avaliam o $m$-commerce sob a perspectiva do usuário (CHONG, 2013; MALIK; KUMRA； SRIVASTAVA， 2013；WU; WANG, 2005; YANG, 2005) e outros sob a perspectiva das empresas (HUANG; QI; DONG, 2007; SHIH; HUANG, 2009). O mcommerce também tem-se mostrado uma oportunidade de inovação por parte das empresas (SCHIERHOLZ; KOLBE; BRENNER, 2005).

Apesar da variedade de estudos, o que se encontra em maior volume na literatura é a pesquisa focada no usuário final. Pesquisas que avaliam o $m$-commerce na condição do ofertante do serviço 
(abordando possíveis benefícios e barreiras que surgem para quem oferece o serviço aos usuários finais) aparentam ser realizadas em menor número, conforme os autores têm observado em pesquisas anteriores.

O relatório Webshoppers, publicado pelo E-bit (2015), que traz dados sobre comércio eletrônico no Brasil, mostra considerável aumento na utilização do $m$ commerce como um canal de compra de produtos (pelos usuários finais). Em janeiro de 2014 apenas 4,8\% das transações de comércio eletrônico eram realizadas por $m$ commerce. Valor que, um ano antes, era de 2,5\%. Já em janeiro de 2015, a participação do $m$-commerce no comércio eletrônico total passou para 9,7\%, considerando ainda que, durante o ano de 2014, o comércio eletrônico como um todo teve aumento real de 26,7\% (E-BIT, 2015). A utilização da telefonia móvel de forma geral também tem apresentado crescimento ininterrupto ao longo dos anos, atingindo a faixa das 280 milhões de linhas ativas no Brasil em dezembro de 2014 , número $3,55 \%$ superior ao registrado 12 meses antes (ANATEL, 2016).

Estes números apontam para a importância e a oportunidade das empresas em inovar com a introdução do $m$ commerce como um novo canal de vendas e/ou relacionamento com os clientes. Dessa forma, considerando esse crescimento no mercado de $m$-commerce, entende-se que é necessária uma visão holística acerca do tema e não somente baseada no usuário final, olhando também para quem oferece o serviço. Assim, as questões que se propôs a responder neste artigo foram: A produção de artigos sobre m-commerce que têm analisado as condições do ofertante do serviço tem crescido nos últimos anos? E o que os trabalhos realizados têm constatado/verificado em relação a quem oferece o serviço?

Sendo assim, esta pesquisa teve como objetivo identificar vantagens $\mathrm{e}$ desafios que as organizações encontram ao oferecerem o serviço de m-commerce.

Após o tópico introdutório, este artigo discorre sobre o que é o $m$-commerce e como ele surgiu, apresenta o método pelo qual esta pesquisa foi desenvolvida, que envolve a descrição de como os artigos foram buscados e filtrados até se chegar aos documentos analisados e, em seguida, inicia-se a apresentação dos resultados. $\mathrm{Na}$ apresentação dos resultados apresentam-se análise bibliométrica do material encontrado e descrição e discussão dos resultados. Considerações finais e sugestões de pesquisas futuras também são realizadas.

\section{M-COMMERCE}

De acordo com Tiwari e Buse (2007, p. 33), "Mobile Commerce é uma transação, envolvendo a transferência de direitos ou propriedades para usar bens e serviços, que 
é iniciada e/ou completada utilizando acesso móvel à internet com a ajuda de um dispositivo eletrônico".

Para deixar essa definição mais clara, os autores elencam alguns pressupostos dos quais partiram: nem todos os dispositivos móveis permitem o acesso móvel à internet e um exemplo disso são os notebooks, pois, mesmo podendo conectarse a uma rede sem fio (wireless) e ser um dispositivo móvel, seu uso é limitado enquanto seu usuário está em movimento; algumas partes da transação podem ser feitas em computador com acesso fixo à internet e, para ser considerado $m$ commerce, pelo menos o início ou o final da transação deve ser realizado por dispositivo eletrônico com acesso móvel à internet; e a transação não precisa envolver um componente monetário, contanto que seja realizada como uma medida de marketing ou um serviço pós-venda (TIWARI; BUSE, 2007). Kourouthanassis e Giaglis (2012) também deixam explícito em seu trabalho que o m-commerce é um serviço acessível por dispositivos móveis enquanto seu usuário está em movimento.

Esse serviço tornou-se possível a partir do desenvolvimento tecnológico da telefonia móvel (JIN; VILLEGAS, 2008), que fez surgir uma ferramenta de marketing com grandes possibilidades como canal de compras (MARTÍN-GUTIÉRREZ; LÓPEZ-CATALÁN; RAMÓN-
JERÓNIMO, 2012). Consequentemente, também com grandes possibilidades como um canal de vendas. Nessa linha, Xu e Yang (2012) afirmam que o m-commerce é útil em diversas partes de um negócio. Entretanto, eles defendem que é importante entender a perspectiva dos usuários do serviço para realizar uma implementação de sucesso dos aplicativos de m-commerce. Para questões mais específicas sobre implementação do serviço, pode-se consultar os trabalhos de revisão de literatura realizados por Ngai e Gunasekaran (2007) e Varnali e Toker (2010), que citam estudos sobre estratégia e modelos de negócio voltados aos serviços móveis (m-commerce e outros). No artigo de Varnali e Toker (2010) também são citados trabalhos que apresentam modelos para melhorar a receita em serviços móveis específicos.

O m-commerce possui algumas características que o diferenciam da tradicional forma de comércio eletrônico. Algumas delas são: possibilidade de serviços baseados na localização do usuário (location-based services) (LEE; HO, 2010; TIWARI; BUSE, 2007); acesso a qualquer hora e em qualquer lugar (THAKUR; SRIVASTAVA, 2013); mobilidade (KOUROUTHANASSIS; GIAGLIS, 2012; LIN et al., 2014; TIWARI; BUSE, 2007); e possibilidade de customização/personalização, que refere-se 
à oferta de produtos e/ou serviços específicos para cada usuário (BARNETT; HODGES; WILSHIRE, 2000; CHONG, 2013 TEZZA; BORNIA; ALBUQUERQUE, 2008).

Referindo-se ao m-commerce como canal de vendas e/ou relacionamento, é possível encontrar na literatura outros termos, tais como m-business. Entretanto, m-business é um conceito de maior abrangência, do qual o m-commerce é apenas uma parte. $\mathrm{O}$ m-business abrange serviços, realizados por meio de tecnologias móveis, como acesso ou compartilhamento de informação, comunicação, transação ( $m$ commerce e outras), e coordenação de atividades (CHU; HUANG, 2008; PIGNEUR, 2002). Apesar do foco aqui não

Figura 1 - Método de seleção de documentos

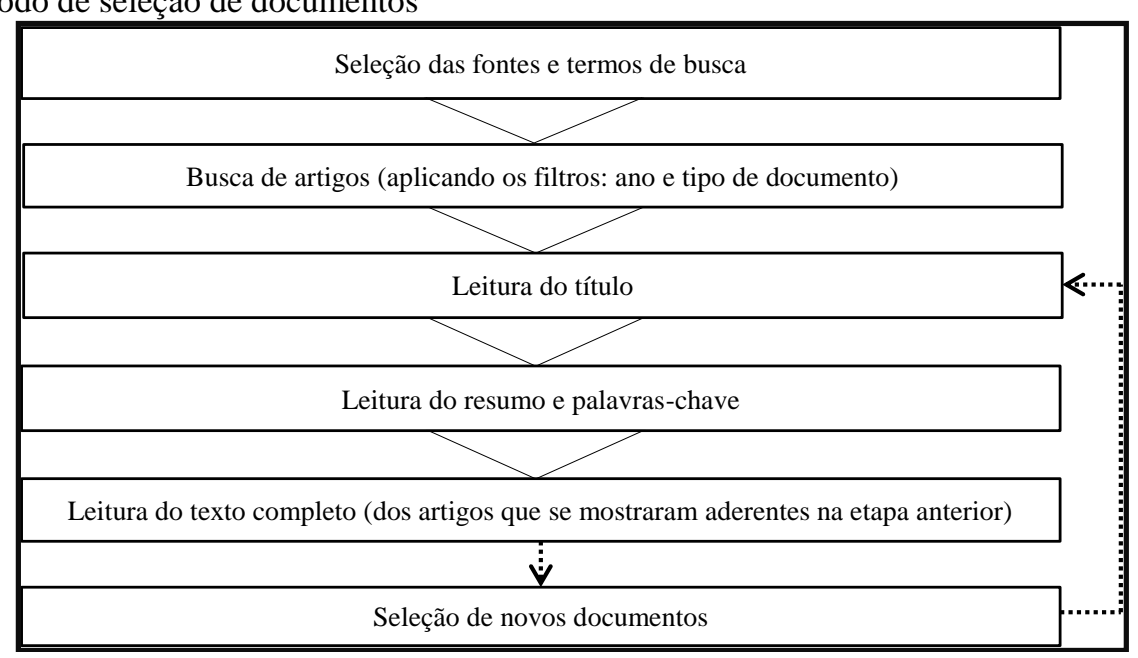

Fonte: Elaborado pelos autores (2015), com base em Villas, Macedo-Soares e Russo (2008).

Para a seleção das fontes e termos de busca, os autores partiram de conhecimento anterior, adquirido em revisão de literatura mais ampla sobre m-commerce. Neste estudo, fez-se necessária uma delimitação ser exatamente o m-business como método de trabalho, o termo business é considerado nesta pesquisa, pois ele também pode ser utilizado para fazer referência a uma transação comercial (PIGNEUR, 2002).

\section{MÉTODO}

Este trabalho trata-se de uma pesquisa documental, qualitativa, realizada com dados secundários. Os dados foram obtidos a partir de uma busca sistemática na literatura, na qual foram buscados artigos que discutem o m-commerce na condição do ofertante do serviço. A busca foi realizada com base em uma adaptação do modelo proposto por Villas, Macedo-Soares e Russo (2008), conforme Figura 1. 
Science Direct, Springer, Web Of Science, e Wiley. Quanto aos termos de busca, utilizaram-se os seguintes: < " $m$ commerce" OR "mobile commerce") AND "case study">; <("m-commerce" OR "mobile commerce") AND ("organi?ation" AND "use")>; <("m-commerce" OR "mobile commerce") AND "innovation">; $<(" m$-commerce" OR "mobile commerce") AND "business use">; <("m-commerce" OR "mobile commerce") AND "seller" >; <("mcommerce" OR "mobile commerce") AND "strategic planning" >; < "m-commerce" OR "mobile commerce") AND "marketing">; e <("m-commerce" OR "mobile commerce") AND "revenue">.

A seleção destes termos foi realizada considerando as seguintes situações: casos sobre organizações que oferecem o serviço de m-commerce, uso do $m$-commerce em organizações, $m$ commerce entendido como uma forma de inovação para quem passa a oferecer o serviço, uso do m-commerce em negócios, m-commerce como uma ferramenta adotada por vendedores, planejamento estratégico que já inclui o m-commerce como canal de vendas e/ou relacionamento, m-commerce como uma ferramenta de marketing e $m$ commerce como uma forma de aumento das receitas. Alguns destes termos foram elencados com base nas revisões de literatura de Ngai e Gunasekaran (2007) e Varnali e Toker (2010) e outros foram estabelecidos a partir de uma revisão mais ampla da literatura sobre m-commerce.

$\mathrm{Na}$ etapa seguinte, buscaram-se os documentos, limitando os termos de busca, apresentados anteriormente, aos campos de título, palavra-chave e resumo, quando a base de dados consultada permitia fazê-lo. Nos demais casos (bases ACM, Springer e Web of Science), buscaram-se os termos de busca no texto completo. Além disso, já na busca, inseriu-se os filtros de "ano de publicação" e "tipo de documento". Com base no tema da pesquisa e na época em que o assunto começou a ganhar destaque, o período de publicação considerado neste trabalho foi de janeiro de 2000 até a data da pesquisa, agosto de 2014. A data de início do período selecionado é a mesma utilizada na revisão de literatura sobre $m$-commerce realizada por Ngai e Gunasekaran (2007). Quanto ao filtro "tipo de documento", buscou-se artigos publicados em anais de congressos (Proceedings) e artigos publicados em revistas científicas (Journals). Tanto os artigos de Journal quanto os de Proceedings foram buscados (apenas) nas bases de dados mencionadas anteriormente, as quais veiculam trabalhos nacionais e internacionais. Buscaram-se documentos escritos nos idiomas português e inglês.

A busca inicial resultou em 1336 referências não duplicadas. Foi utilizado o software EndNote $X 7^{\circledR}$ para organização do 
material. O passo seguinte foi a leitura dos títulos para eliminar referências não aderentes ao objetivo da pesquisa. Dos 257 artigos que restaram, leram-se as palavraschave e o resumo e constatou-se que apenas 22 poderiam estar relacionados ao que se buscava. Entretanto, ao ler e analisar os textos completos, foram eliminados mais seis artigos, que não atendiam aos objetivos da pesquisa. Cabe destacar que somente obteve-se acesso aos artigos disponíveis em bases de dados assinadas pela CAPES.

O próximo passo foi a busca por novos documentos, seguindo o método de Chang, Cheung e Lai (2005). Para tal, selecionou-se a revista Industrial Management \& Data Systems, pois é uma das revistas encontradas na busca inicial e que tem publicado vários estudos sobre $m$ commerce. Nenhum artigo novo foi encontrado. Desse modo, com base nos 16 artigos restantes, foi realizada análise bibliométrica e análise descritiva de conteúdo (ALVES, 2011), buscando apresentar os principais resultados atingidos pelos estudos, acompanhada de uma discussão acerca dos resultados.

Apesar de na revisão manual em uma das revistas não terem sido encontrados novos documentos, não é descartada a possibilidade de que os termos de busca utilizados tenham ignorado outros estudos aderentes ao objetivo da pesquisa. Portanto, deve-se considerar como uma possível limitação o fato de nem todos os artigos relacionados ao objetivo do estudo terem sido encontrados.

\section{ANÁLISE DOS RESULTADOS}

Neste tópico, buscou-se contabilizar e classificar as publicações encontradas sobre o tema de interesse deste artigo e realizar uma análise sobre os resultados encontrados. A bibliometria mostra que há dezesseis publicações acadêmicas sobre $m$ commerce cujo foco de análise é o ofertante do serviço. A Tabela 1 traz uma classificação das publicações encontradas no período considerado. 
Tabela 1 - Classificação e quantidade de artigos encontrados no período analisado

\begin{tabular}{|c|c|}
\hline Classificação & $N$ \\
\hline Total artigos & 16 \\
\hline Journal & 9 \\
\hline Proceeding & 7 \\
\hline \multicolumn{2}{|l|}{ Tipo de Pesquisa } \\
\hline Estudo de caso & 3 \\
\hline Estudo de caso / proposta ${ }^{a}$ & 3 \\
\hline Revisão de Literatura & 2 \\
\hline Revisão de Literatura / estudo de caso & 1 \\
\hline Survey com empresas & 2 \\
\hline Teórica & 4 \\
\hline Teórica / proposta ${ }^{a}$ & 1 \\
\hline \multicolumn{2}{|l|}{ Ano } \\
\hline 2002 & 2 \\
\hline 2003 & 2 \\
\hline 2005 & 1 \\
\hline 2007 & 1 \\
\hline 2008 & 1 \\
\hline 2009 & 3 \\
\hline 2010 & 3 \\
\hline 2012 & 2 \\
\hline 2013 & 1 \\
\hline \multicolumn{2}{|l|}{ País ${ }^{b}$} \\
\hline Austrália & 1 \\
\hline China & 1 \\
\hline Espanha & 1 \\
\hline Estados Unidos & 3 \\
\hline Estados Unidos / Austrália & 1 \\
\hline Estados Unidos / China & 1 \\
\hline Grécia & 1 \\
\hline Noruega & 1 \\
\hline Nova Zelândia & 1 \\
\hline República Tcheca & 1 \\
\hline Suíça & 2 \\
\hline Taiwan & 2 \\
\hline
\end{tabular}

\section{Tipo de Empresa}

Seguros

Tecnologia da Informação

Telecomunicações

Serviço de localização on-line

Serviços móveis

Marketing móvel

Diversas (Survey)

Plano de saúde 
Tabela 1 - Classificação e quantidade de artigos encontrados no período analisado (cont.)

\begin{tabular}{ll}
\hline \multicolumn{1}{c}{ Classificação } & $n$ \\
\hline Local de publicação & 1 \\
\hline Issues e Trends of Information Technology Management in Contemporary Organizations & 1 \\
\hline Journal of the Academy of Marketing Science & 1 \\
\hline International Journal of Information Technology \& Decision Making & 1 \\
\hline $\begin{array}{l}\text { Proceedings of the 14th International Workshop on Database e Expert Systems } \\
\text { Applications }\end{array}$ & 1 \\
\hline Wirtschaftsinformatik & 1 \\
\hline $\begin{array}{l}\text { 3rd International Conference on Wireless Communications, Networking e Mobile } \\
\text { Computing }\end{array}$ & 1 \\
\hline Journal of Business Strategies & 1 \\
\hline Fifth Advanced International Conference on Telecommunications & 1 \\
\hline $\begin{array}{l}\text { Proceedings of the 2009 Sixth International Conference on Information Technology: } \\
\text { New Generations }\end{array}$ & 1 \\
\hline Telecommunications Policy & 1 \\
\hline Electronic Markets & 1 \\
\hline Journal of International Technology e Information Management & 1 \\
\hline $\begin{array}{l}\text { Proceedings of the International Conference on Communication e Management in } \\
\text { Technological Innovation e Academic Globalization }\end{array}$ & 1 \\
\hline Industrial Management \& Data Systems & 1 \\
\hline Journal of Electronic Commerce in Organizations & 1 \\
\hline Proceedings of the 2013 International Academic Workshop on Social Science & 1 \\
\hline Por Propostantense a proposicaro de um mode que pode ser utizado entudos futr. & 1 \\
\hline
\end{tabular}

Nota. ${ }^{\text {a }}$ Por Proposta entende-se a proposição de um modelo que pode ser utilizado em estudos futuros.

${ }^{\mathrm{b}}$ Considerou-se o país de origem dos dados analisados. Nos casos de estudos teóricos ou revisão de literatura considerou-se a nacionalidade dos autores.

${ }^{\mathrm{c}}$ Estudos teóricos (baseados em teorias) e revisão de literatura (levantamento da literatura).

Fonte: Elaborado pelos autores (2015).

A partir dos resultados apresentados na Tabela 1, foi possível realizar algumas considerações. Primeiramente, verifica-se que sete dos artigos encontrados são artigos publicados em anais de congressos (Proceedings). Isso indica caráter prático das publicações. Além disso, dentre os artigos que adotam o estudo de caso ou survey como método de pesquisa, apenas quatro são artigos publicados em revistas acadêmicas.

Em segundo lugar, quanto ao tipo de dados utilizados, nota-se que apenas nove artigos reportam pesquisas baseadas em dados primários. Isso demonstra que ainda é baixo o número de estudos que busca avaliar se a literatura existente sobre $m$ commerce é de fato aplicável à prática das empresas. Um número maior de estudos baseados em dados primários poderia revelar se a literatura é de fato aplicável em sua totalidade para qualquer ramo empresarial, ou se existem especificidades no $m$-commerce para cada tipo de empresa e, se existem, quais são elas.

Quanto ao ano de publicação, verifica-se que os primeiros estudos foram publicados em 2002. Desde então, a produção anual de artigos sobre o tema tem oscilado. Nos anos de 2004, 2006, 2011 e 
2014 nenhum artigo foi publicado. Em contrapartida, nos anos de 2009 e 2010 houve um pico de três publicações em cada ano. Não se encontrou uma razão explícita que justifique esse resultado.

Estados Unidos é o país mais presente nas publicações sobre $m$ commerce com o tema pesquisado. Cinco dos estudos publicados foram desenvolvidos no país ou foram desenvolvidos em parceria com autores estadunidenses (Somente foram considerados como estudos desenvolvidos em parceria os que utilizaram dados secundários - estudos teóricos e revisão de literatura. Nos estudos baseados em dados primários que tiveram autores de diferentes países, foi considerado somente o país no qual os dados foram coletados). Em seguida aparecem Austrália, Suíça e Taiwan, com dois estudos cada ou em parceria.

Nos demais países apresentados, apenas um estudo foi desenvolvido no período analisado. Além de ter a maior quantidade de estudos, os EUA também têm a maior quantidade de estudos publicados em revistas acadêmicas, sendo três deles de autores (ou em parceria com) estadunidenses. Em seguida aparece a Austrália, com dois estudos publicados em revistas acadêmicas. Os outros países possuem no máximo uma publicação em revistas e as demais são publicações em anais de congressos. Em relação ao Brasil, nenhum estudo foi encontrado.

Nos estudos que utilizaram dados primários, verificou-se que não existe foco em um único tipo de empresa. Cada um deles foi desenvolvido em um contexto diferente. Considerando esse resultado, este artigo também apresenta algumas semelhanças em relação ao m-commerce entre diferentes tipos de negócios.

Já em relação ao local de publicação dos estudos, observa-se que não existe uma revista específica na qual são publicadas as pesquisas com o foco tratado neste artigo. Cada um dos trabalhos analisados está publicado em diferentes locais.

A partir da revisão de literatura e analisando o conteúdo dos artigos selecionados para este estudo, verificou-se que é possível reunir os resultados reportados nestes artigos em quatro grupos: fatores de influência à oferta do serviço de m-commerce; benefícios e efeitos obtidos com a oferta do serviço de m-commerce; barreiras para a oferta do serviço de $m$ commerce; e fatores críticos de sucesso na oferta do serviço de m-commerce. A nomenclatura atribuída a cada um dos grupos, apresentados a seguir, baseou-se em seu conteúdo. 


\subsection{Fatores que influenciam a decisão de} oferecer o serviço de m-commerce

Dos dezesseis trabalhos em análise, apenas três apresentam discussão acerca de fatores que podem impactar na intenção das empresas/organizações de oferecerem o serviço de m-commerce. Os resultados seguem apresentados no Quadro 1.

Quadro 1 - Fatores de influência à oferta do serviço de m-commerce
\begin{tabular}{|l|c|c|}
\hline \multicolumn{1}{|c|}{ O que pode levar as organizações a oferecerem o m-commerce } & $\begin{array}{c}\text { Tipo de } \\
\text { estudo }\end{array}$ & Referência \\
\hline $\begin{array}{l}\text { Busca por melhorar o relacionamento com os consumidores e aumentar } \\
\text { as receitas. }\end{array}$ & Estudo de caso & $\begin{array}{c}\text { Huang, Qi e } \\
\text { Dong (2007) }\end{array}$ \\
\hline $\begin{array}{l}\text { Busca por manter a vantagem competitiva; utilizar o m-commerce como } \\
\text { forma de integrar os processos empresariais para melhorar o } \\
\text { desempenho; e proporcionar informação aos clientes. }\end{array}$ & $\begin{array}{c}\text { Revisa de } \\
\text { literatura com } \\
\text { estudo de caso }\end{array}$ & $\begin{array}{c}\text { Shih e Huang } \\
(2009)\end{array}$ \\
\hline $\begin{array}{l}\text { Ambiente político e legal; políticas organizacionais; infraestrutura de } \\
\text { TIC; cultura organizacional; vantagens relativas; usabilidade percebida; } \\
\text { facilidade de uso percebida; complexidade; compatibilidade; capacidade } \\
\text { da inovação de melhorar o desempenho individual; suporte da cúpula } \\
\text { gerencial; segurança das informações e propriedade intelectual; } \\
\text { confiança; fatores sociais; e normas subjetivas. }\end{array}$ & $\begin{array}{c}\text { Revisão de } \\
\text { literatura }\end{array}$ & $\begin{array}{c}\text { Houghton e } \\
\text { Sanzogni } \\
(2012)\end{array}$ \\
\hline
\end{tabular}

Fonte: Elaborado pelos autores (2015).

Com base nos resultados, verifica-se semelhança entre a abordagem de Huang, Qi e Dong (2007) e a abordagem de Shih e Huang (2009). Essas duas pesquisas apontam como fatores impulsionadores a busca por manter vantagem competitiva e a possibilidade de utilizar o m-commerce como forma de integrar processos empresariais, visando melhorar $\mathrm{o}$ desempenho e proporcionar informação aos clientes. Nota-se que esses fatores são voltados às vantagens que a adoção do $m$ commerce pode trazer para as empresas e os autores enfatizam que esses pontos causam impacto positivo na intenção de adoção do serviço.

Por outro lado, Alfahl, Houghton e Sanzogni (2012) são neutros em sua abordagem. Como pode ser visto no Quadro 1, esses autores apresentam diversos fatores, mas não apontam se são fatores de impacto positivo ou de impacto negativo. $\mathrm{O}$ que é possível perceber é que eles apresentam fatores tanto do ambiente interno quanto do ambiente externo, além de fatores relacionados à percepção dos indivíduos que trabalham na empresa. Em relação ao ambiente externo, os autores citam o ambiente político e legal e fatores sociais. Já em relação ao ambiente interno, são apontados: as políticas organizacionais; a cultura organizacional; as vantagens relativas à adoção, que são apresentadas no sentido de avaliar se as vantagens apontadas realmente trarão benefícios para a organização; complexidade; compatibilidade; capacidade da inovação de melhorar o desempenho individual; suporte da alta administração; e normas subjetivas. A infraestrutura de TIC (Tecnologia da 
Informação e Comunicação) é apresentada pelos autores tanto como uma variável de impacto do ambiente interno quanto externo, pois é necessário que exista uma infraestrutura de TIC disponível fora da empresa para oferecer o serviço aos usuários e é necessário que a empresa também tenha uma infraestrutura de TIC interna para oferecer o serviço em questão. Por fim, como fatores relacionados à percepção dos indivíduos, destacam-se a usabilidade, a facilidade de uso, segurança da informação e propriedade intelectual, e a confiança.

Como é possível perceber, os fatores apontados por Alfahl, Houghton e Sanzogni (2012) podem exercer influência tanto positiva quanto negativa na intenção de uma organização oferecer o serviço de $m$ commerce. Entretanto, esses fatores podem ser tomados como base para desenvolver estudos que busquem avaliar o tipo de influência que cada um deles exerce na prática.
Com base no que esses estudos apresentam, verifica-se que os principais motivos que levam à oferta do serviço de $m$ commerce são relacionados à elevação das receitas da organização, melhoria do relacionamento com seus clientes, manutenção da vantagem competitiva e, com foco apenas interno, um meio de integração dos processos empresariais. De forma geral, pode-se dizer que a principal influência à intenção de oferecer o serviço de $m$-commerce é o interesse de melhorar a situação financeira da organização.

\subsection{Benefícios e efeitos obtidos com a oferta do serviço de m-commerce}

Dentre os estudos analisados, onze apresentam benefícios e/ou efeitos que a oferta do serviço de m-commerce gera, ou pode gerar, nas empresas que o oferecem. Os resultados seguem apresentados no Quadro 2.

Quadro 2 - Benefícios / Efeitos obtidos com a oferta do serviço de $m$-commerce

\begin{tabular}{|c|c|c|}
\hline Benefícios / Efeitos obtidos com a oferta do serviço de $m$-commerce & $\begin{array}{l}\text { Tipo de } \\
\text { estudo }\end{array}$ & Referência \\
\hline $\begin{array}{l}\text { Benefícios: Utilizar da localização do usuário para comunicar-lhe um } \\
\text { preço específico (oferta exclusiva a um usuário em particular } \\
\text { comunicando-lhe o preço por meio do dispositivo móvel); pode-se } \\
\text { considerar o local e o tempo em que os consumidores se encontram. } \\
\text { Efeitos: Precificação dos produtos no varejo e competição tendem a ser } \\
\text { mais complexos; numa perspectiva de marketing, o m-commerce } \\
\text { promete maior liberdade de precificação quando se formula uma } \\
\text { estratégia de preço no varejo. }\end{array}$ & Teórico & $\begin{array}{c}\text { Balasubramanian, } \\
\text { Peterson e } \\
\text { Jarvenpaa (2002) }\end{array}$ \\
\hline
\end{tabular}


Quadro 2 - Benefícios / Efeitos obtidos com a oferta do serviço de m-commerce (cont.)

\begin{tabular}{|c|c|c|}
\hline Benefícios / Efeitos obtidos com a oferta do serviço de m-commerce & $\begin{array}{l}\text { Tipo de } \\
\text { estudo }\end{array}$ & Referência \\
\hline $\begin{array}{l}\text { Benefícios: Criação de uma zona de comércio sem fio (shopping } \\
\text { virtual); "deslocamento" da loja para onde seu contato com os clientes } \\
\text { for maior; personalização - baseada na localização do usuário e no nível } \\
\text { psicológico do indivíduo (monitoramento das atividades do usuário gera } \\
\text { dados que aprimoram os filtros, permitindo oferecer-lhe produtos e } \\
\text { serviços nos quais ele realmente possa estar interessado); utilidade } \\
\text { promocional - divulgar promoções com base nas zonas criadas e na } \\
\text { personalização; nível de interação entre vendedores e compradores - o } \\
\text { m-commerce pode agilizar a interação entre vendedores e compradores, } \\
\text { preencher as lacunas deixadas pelo e-commerce, além de diminuir o } \\
\text { tempo entre a propaganda e a operação de venda. } \\
\text { Efeitos: Fornecedores podem ser forçados a estenderem suas operações } \\
\text { para regiões maiores para aumentar a interação com os clientes - essa } \\
\text { expansão deve ser direcionada no sentido de estar presente em mais de } \\
\text { uma zona, não no sentido de ter uma forte presença em apenas uma zona } \\
\text { em particular. }\end{array}$ & Teórico & Jukic et al. (2002) \\
\hline $\begin{array}{l}\text { Benefícios: Usuário sempre conectado; possibilidade de } \\
\text { reconhecimento da localização do usuário; serviços personalizados } \\
\text { (baseados na localização e no comportamento do usuário); geração de } \\
\text { valor para a empresa; e serviços com valor adicionado (por meio da } \\
\text { criação de novos modelos de negócio ou modificação dos modelos } \\
\text { existentes). }\end{array}$ & Teórico & $\begin{array}{l}\text { Yeo e Huang } \\
\quad(2003)\end{array}$ \\
\hline Benefícios: Demonstrar a capacidade de inovação. & $\begin{array}{l}\text { Revisão de } \\
\text { literatura }\end{array}$ & $\begin{array}{l}\text { Schierholz, Kolbe } \\
\text { e Brenner (2005) }\end{array}$ \\
\hline Benefícios: Localização; personalização; omnipresença; e pontualidade. & $\begin{array}{l}\text { Revisão de } \\
\text { literatura com } \\
\text { estudo de caso } \\
\end{array}$ & $\begin{array}{l}\text { Huang, Qi e Dong } \\
\qquad(2007)\end{array}$ \\
\hline $\begin{array}{l}\text { Benefícios: Marketing direcionado com base na localização e } \\
\text { características do usuário. }\end{array}$ & Teórico & Clarke (2008) \\
\hline \multirow{2}{*}{$\begin{array}{l}\text { Benefícios: Personalização de serviços baseada na localização do } \\
\text { usuário (location-based) e no contexto (context-awareness). }\end{array}$} & $\begin{array}{l}\text { Estudo de caso } \\
\text { com proposta }\end{array}$ & Georgiadis (2009) \\
\hline & Estudo de caso & $\begin{array}{c}\text { Sharma e } \\
\text { Gutiérrez (2010) }\end{array}$ \\
\hline $\begin{array}{l}\text { Benefícios: Envio de mensagens personalizadas (marketing) aos } \\
\text { usuários com base nos hábitos de navegação e nos padrões de compra. }\end{array}$ & $\begin{array}{l}\text { Estudo de caso } \\
\text { com proposta }\end{array}$ & Lee e Ho (2010) \\
\hline $\begin{array}{l}\text { Benefícios: Marketing baseado na localização (location-based } \\
\text { marketing) está exercendo grande papel no m-commerce relacionado às } \\
\text { táticas de marketing dos principais varejistas; Inovação em marketing; } \\
\text { redução de custos; e oportunidade de atingir melhores consumidores. }\end{array}$ & Survey & $\begin{array}{l}\text { Tomášková } \\
\text { (2010) }\end{array}$ \\
\hline $\begin{array}{l}\text { Benefícios: Geração de dados em massa (pelo m-commerce) combinado } \\
\text { com Business Intelligence (BI) gera: informação confiável; operação } \\
\text { personalizada; informação instantânea sobre o desempenho; descrição e } \\
\text { classificação do comportamento dos consumidores; geração de } \\
\text { marketing personalizado (a partir do BI); e oferta de informação e } \\
\text { serviços customizados para melhorar a experiência do usuário. }\end{array}$ & $\begin{array}{l}\text { Teórico com } \\
\text { proposta }\end{array}$ & $\begin{array}{l}\text { Gan, Tu e Wang } \\
\text { (2013) }\end{array}$ \\
\hline
\end{tabular}

Fonte: Elaborado pelos autores (2015).

Com base nos resultados, observa-se que a possibilidade de uma empresa utilizar as informações de localização aliadas ao contexto dos usuários de dispositivos móveis é apontada em vários estudos como um dos benefícios da oferta do serviço de m-commerce. As empresas que oferecem o serviço podem fazer uso desse tipo de informação para aprimorar suas ofertas a clientes específicos e, assim, melhorar o relacionamento com os mesmos. 
Diferentemente da ideia de localização e contexto, verifica-se que outros autores trabalham a ideia de localização aliada a características e hábitos do usuário, como pode ser verificado no Quadro 2. Especificamente sobre marketing direcionado, Clarke (2008) afirma que é possível realiza-lo porque os dispositivos móveis têm, normalmente, apenas um único usuário. Apesar da delimitação do autor ao marketing, entende-se que isso é válido para todos os serviços oferecidos via dispositivo móvel de comunicação.

Apesar da combinação realizada entre os fatores localização e contexto do usuário e os fatores localização e características dos usuários que os trabalhos apresentados, alguns autores também consideram que a localização pode ser um fator isolado, ou seja, não aliado a outros fatores de personalização. Um desses trabalhos é o de Jukic et al. (2002), que, apesar de apresentarem a ideia de localização combinada com características do usuário, também falam sobre a criação de uma zona de comércio eletrônico, similar a um shopping virtual, que se baseia na localização dos usuários. Segundo os autores, essa zona de comércio virtual permite às lojas estarem em, ou irem para, locais em que seu contato com os clientes será maior, aumentando as possibilidades de venda. $\mathrm{O}$ outro trabalho que aposta na localização como fator isolado (não aliado a outros fatores de personalização) é o de Tomášková (2010), que afirma que o marketing baseado na localização está exercendo grande papel no m-commerce relacionado às táticas de marketing dos principais varejistas situados na República Tcheca.

Assim como os autores que apresentam a localização como um fator não combinado, há também os que trazem a personalização baseada nas características do usuário como um fator isolado, ou seja, a personalização baseia-se apenas nas características do usuário. Um desses trabalhos é o de Lee e Ho (2010), que apresentam, dentre os demais, o caso da empresa Chunghwa, que envia mensagens de marketing personalizado aos usuários de dispositivos móveis e, como critério de personalização, utiliza informações como hábitos de navegação e padrões de compra. E outro desses trabalhos é o de Gan, Tu e Wang (2013). Esses autores discutem sobre a integração dos sistemas de BI (Business Intelligence - data warehouse, On-Line Analysis processing (OLAP) e data mining) com o m-commerce.

Com base nos dados obtidos a partir do m-commerce, os sistemas de BI podem descrever e classificar o comportamento dos consumidores, gerando marketing personalizado, e podem promover informação customizada e serviços para melhorar a experiência do usuário. Estes 
dois artigos (GAN; TU; WANG, 2013; LEE; HO, 2010) apontam que a personalização baseada unicamente nas características dos usuários também pode gerar resultados satisfatórios. O que se propõe nesse ponto é que isso pode depender do tipo de negócio que a empresa desenvolve.

$\mathrm{O}$ que foi apresentado até aqui refere-se basicamente à oferta de produtos, serviços e marketing personalizado, considerando informações como localização, características e comportamento do usuário. Entretanto, no Quadro 2 ainda podem ser visualizados outros benefícios apontados nos trabalhos analisados. O referido quadro também apresenta alguns efeitos que os benefícios apontados podem gerar. Apesar dos resultados apresentados serem restritos aos casos analisados, acredita-se que todos os benefícios e efeitos da adoção do $m$ commerce citados na literatura são importantes para qualquer tipo de negócio, mesmo que cada um tenha suas particularidades.

De modo geral, pode-se dizer que o grande benefício de se oferecer o serviço de m-commerce é dispor das informações dos clientes, tanto relacionadas à localização, como caraterísticas pessoais, que são fundamentais para a oferta de produtos e serviços personalizados e melhorar o relacionamento com os clientes. No entanto, é compromisso básico da organização garantir o sigilo das informações coletadas, as quais não devem ser utilizadas para nenhuma finalidade além da que o usuário tenha concordado, ou tenha ciência.

\subsection{Barreiras para a oferta do serviço de m-commerce}

Apenas cinco dos dezesseis estudos analisados citam barreiras para as empresas oferecerem o serviço de m-commerce. Dentre eles, observa-se que quatro adotam, unicamente ou combinado, o estudo de caso como método de pesquisa, ou seja, em algum momento as análises desses quatro artigos valem-se de dados primários, buscados na vivência organizacional. Os resultados seguem apresentados no Quadro 3. 
Quadro 3 - Barreiras para a oferta do serviço de $m$-commerce

\begin{tabular}{|c|c|c|}
\hline Barreiras para a oferta do serviço de $m$-commerce & Tipo de estudo & Referência \\
\hline $\begin{array}{l}\text { Diferentes padrões de tecnologia; segurança; e baixa velocidade de } \\
\text { conexão. }\end{array}$ & Teórico & $\begin{array}{c}\text { Yeo e } \\
\text { Huang } \\
(2003) \\
\end{array}$ \\
\hline $\begin{array}{l}\text { Diferentes padrões de tecnologia; diferentes sistemas operacionais; } \\
\text { segurança; baixa velocidade de conexão; interfaces gráficas não adaptadas } \\
\text { para telas pequenas; custo da conexão sem fio; estratégias de marketing } \\
\text { inadequadas; nível de conforto do usuário; questões culturais e } \\
\text { comportamento do consumidor. }\end{array}$ & $\begin{array}{c}\text { Revisão de } \\
\text { literatura com } \\
\text { estudo de caso }\end{array}$ & $\begin{array}{l}\text { Huang, Qi e } \\
\text { Dong (2007) }\end{array}$ \\
\hline $\begin{array}{l}\text { Rivalidade entre regimes de conhecimento (regime tecnológico e regime } \\
\text { orientado para os negócios); complexidade tecnológica subestimada; } \\
\text { incerteza sobre as necessidades do mercado e dos clientes (também em } \\
\text { relação à qualidade do serviço); modelo de negócios inadequado; falta de } \\
\text { convergência entre instituições - o desenvolvimento do m-commerce } \\
\text { requer a convergência de várias instituições e mecanismos que atualmente } \\
\text { encontram-se separados a autônomos, logo, políticas e estratégias } \\
\text { precisam ser desenvolvidas para superar isso. }\end{array}$ & Estudo de caso & $\begin{array}{c}\text { Godoe e } \\
\text { Hansen } \\
(2009)\end{array}$ \\
\hline $\begin{array}{l}\text { Lei da privacidade impede serviços baseados na localização (no caso do } \\
\text { país estudado). }\end{array}$ & $\begin{array}{l}\text { Estudo de caso } \\
\text { com proposta }\end{array}$ & $\begin{array}{l}\text { Lee e Ho } \\
(2010)\end{array}$ \\
\hline $\begin{array}{l}\text { Modelos de negócio inadequado (modelos orientados para o e-commerce } \\
\text { não são necessariamente modelos para o } m \text {-commerce, pois a cadeia de } \\
\text { valor para o } m \text {-commerce é muito mais complexa); diferentemente do } e \text { - } \\
\text { commerce, no } m \text {-commerce o usuário não está livre para acessar Internet, } \\
\text { ele depende de empresas de telecomunicação móvel e de serviços de } \\
\text { dados, além disso, o usuário é cobrado pelo volume de dados que trafegam } \\
\text { em sua conexão; custo do serviço de } m \text {-commerce é ditado pelo provedor } \\
\text { de comunicação móvel. }\end{array}$ & Estudo de caso & $\begin{array}{c}\text { Sharma e } \\
\text { Gutiérrez } \\
(2010)\end{array}$ \\
\hline
\end{tabular}

Fonte: Elaborado pelos autores (2015).

Com base nas informações do

Quadro 3, verifica-se que algumas barreiras apontadas tratam-se de casos específicos, como é o caso do estudo de Lee e Ho (2010). No entanto, outros estudos apresentam barreiras mais gerais, que podem, inclusive, atravessar fronteiras. Um exemplo disso são as barreiras culturais e de comportamento do consumidor (HUANG; QI; DONG, 2007). Os autores citam o caso dos EUA e do Japão, destacando que os norte-americanos não gostam de telas pequenas, enquanto os japoneses são adeptos a telas pequenas e gostam de limitação de espaço na tela.

Outras barreiras encontradas nos estudos dizem respeito a especificidades da comunicação móvel. Sharma e Gutiérrez (2010), por exemplo, afirmam que o $\mathrm{m}$ commerce não é um mercado aberto, mas um mercado controlado pelos provedores de comunicação móvel. Além destes, o Quadro 3 ainda traz outros fatores que dificultam a oferta do $m$-commerce.

Com base nos estudos analisados, observa-se que as principais barreiras são relacionadas à diversidade de padrões de tecnologia, conexão móvel e, internamente à organização ofertante, modelos de negócio inadequados, que não comportam a oferta do serviço. Em resumo, as barreiras apresentadas pela literatura sugerem que a oferta do serviço de m-commerce não é tarefa simples e que requer uma interação 
mínima entre os atores envolvidos. No entanto, considera-se importante que barreiras e benefícios sempre sejam analisados lado a lado, pois assim será possível verificar mais facilmente se o esforço necessário será compensado. Uma análise prévia bem-feita pode poupar a organização de incorrer em perdas com as quais possa não conseguir arcar.

\subsection{Fatores críticos de sucesso na oferta} do serviço de $m$-commerce

Tratando-se de fatores críticos de sucesso, seis foram os artigos encontrados que debatem esses fatores. Cabe destacar que cinco dessas pesquisas buscaram dados primários, ou seja, em algum momento desses artigos os resultados são baseados em dados reais, buscados na prática organizacional. Os resultados seguem apresentados no Quadro 4.

Quadro 4 - Fatores críticos de sucesso na oferta do serviço de $m$-commerce

\begin{tabular}{|c|c|c|}
\hline Fatores Críticos de Sucesso & Tipo de estudo & Referência \\
\hline $\begin{array}{l}\text { A adoção da tecnologia deve considerar se realmente há mercado } \\
\text { suficiente para justificar a adoção da tecnologia em questão; deve } \\
\text { haver um equilíbrio entre possibilidades técnicas e otimização } \\
\text { econômica. }\end{array}$ & Teórico & $\begin{array}{l}\text { Balasubramanian, } \\
\quad \text { Peterson e } \\
\text { Jarvenpaa (2002) }\end{array}$ \\
\hline $\begin{array}{l}\text { M-commerce deve ser parte da estratégia corporativa e estar } \\
\text { embutido na estratégia de marketing; utilização de componentes } \\
\text { existentes e implementação baseada em produtos-padrão para manter } \\
\text { os custos baixos; cadeia de valor exerce um papel importante (desde } \\
\text { que a terceirização seja um facilitador para economia de tempo e } \\
\text { custo); simplicidade; e desempenho. }\end{array}$ & Estudo de caso & $\begin{array}{l}\text { Reichold et al. } \\
\text { (2003) }\end{array}$ \\
\hline $\begin{array}{l}\text { Iniciar com uma estratégia de necessidade, não com uma estratégia } \\
\text { tecnológica; desenvolver uma estratégia eletrônica que complemente } \\
\text { a estratégia empresarial; entender e focar os clientes certos; utilizar } \\
\text { os recursos sabiamente; garantir segurança e credibilidade aos } \\
\text { clientes; proporcionar uma experiência completa aos clientes; } \\
\text { entregar serviços personalizados; proporcionar conveniência aos } \\
\text { clientes; e ter um modelo de negócio adequado. }\end{array}$ & $\begin{array}{l}\text { Revisão de } \\
\text { literatura com } \\
\text { estudo de caso }\end{array}$ & $\begin{array}{l}\text { Huang, Qi e } \\
\text { Dong (2007) }\end{array}$ \\
\hline $\begin{array}{l}\text { Tecnológico - Estratégia do 'produto' móvel; qualidade da } \\
\text { tecnologia de informação; hardware; software; e capacidade da } \\
\text { conexão sem fio. } \\
\text { Organizacional - Vantagem competitiva; apoio à gestão; cultura e } \\
\text { ambiente. } \\
\text { Social - Influência social; Gestão do relacionamento com o } \\
\text { consumidor (CRM). } \\
\text { Pessoal - Comportamento e auto eficácia; estratégias de tarefa; e } \\
\text { consideração de restrições pessoais. }\end{array}$ & Estudo de caso & $\begin{array}{l}\text { Shih e Huang } \\
\quad(2009)\end{array}$ \\
\hline
\end{tabular}


Quadro 4 - Fatores críticos de sucesso na oferta do serviço de $m$-commerce (cont.)

\begin{tabular}{|l|c|c|}
\hline \multicolumn{1}{|c|}{ Fatores Críticos de Sucesso } & Tipo de estudo & Referência \\
\hline Relacionados ao serviço: Proporcionar a melhor experiência ao & & \\
usuário em questões de facilidade de uso, acessibilidade, serviços \\
oferecidos (integralidade das atividades de cadeia de valor), \\
proposição de valor aos clientes; opções de pagamento facilitadas; \\
habilidade do modelo de negócios mudar e evoluir com o tempo - \\
dinamicidade; empresa deve ajustar-se a diferentes parâmetros de \\
mercado; modelo de negócio deve ser centrado no usuário \\
(colocando o potencial cliente em primeiro lugar); interface boa, fácil \\
de usar e acessível pode ser um fator decisivo para o sucesso do \\
modelo de negócios, pois quanto mais utilizável é a interface, mais & Estudo de caso & Gutiérrez (2010) \\
viável torna-se o modelo de negócios e maior é a usabilidade & & \\
percebida uma boa interface pode elevar significativamente a & & \\
proposição de valor). & & \\
Relacionados à organização: Interesse em colaborar e formar & & \\
parcerias com outros atores da indústria; estrutura de custos & & \\
adequada e padronizada, juntamente a um modelo de organização; & & \\
bom e suficiente retorno sobre o investimento para cada parceiro de & & \\
negócio; colaboração e parcerias; e capacidade de resposta às & & \\
tendências de mercado. & & \\
\hline $\begin{array}{l}\text { Conhecer a carteira de clientes; fazer uma segmentação adequada } \\
\text { para personalizar o conteúdo; adaptar as estratégias para uso de } \\
\text { dispositivos móveis para comércio, com a receptividade dos clientes; } \\
\text { considerar a importância da análise de viabilidade do m-commerce } \\
\text { para cada setor e cada caso em específico; considerar o ajuste } \\
\text { tecnológico requerido; analisar a receptividade do m-marketing e } \\
\text { compras à distância pela carteira de clientes da empresa. }\end{array}$ & Survey & Catalán e Ramón- \\
\hline
\end{tabular}

Fonte: Elaborado pelos autores (2015).

Com base no Quadro 4, verifica-se que um fator apontado em pesquisas diversas diz respeito à importância de questões estratégicas. Reichold et al. (2003), por exemplo, constataram que, durante o processo de implantação do $m$ commerce, a utilização de componentes existentes e implantação baseada em produtos-padrão foram fundamentais para manter os custos baixos, fazendo com que fatores como simplicidade e desempenho tenham sido importantes para o sucesso do m-commerce no caso analisado.

Por sua vez no estudo de Martín, López-Catalán e Ramón-Jerónimo (2012), concluiu-se que competência tecnológica, adaptação das atividades da empresa ao $m$ - commerce e valor percebido pelo consumidor exercem efeito direto e positivo no desempenho do m-commerce. No mesmo estudo, constatou-se que pressão competitiva não exerce efeito sobre o desempenho do serviço em questão. Entretanto, a vantagem competitiva é um fator crítico de sucesso para o m-commerce (SHIH; HUANG, 2009). A pressão competitiva refere-se à pressão exercida pela concorrência, enquanto que a vantagem competitiva refere-se ao fato da empresa estar à frente da concorrência.

Fatores tecnológicos também são citados como fatores críticos de sucesso, principalmente no que diz respeito a uma estratégia de produto móvel. A organização 
precisa considerar a qualidade da tecnologia de informação utilizada, as restrições que podem ser encontradas em questões de hardware e software e as restrições que podem ser impostas pela conexão sem fio (SHIH; HUANG, 2009).

Além das variáveis relacionadas à estrutura e a estratégias da organização em si, há também uma série de fatores relacionados ao cliente, que também devem ser considerados para uma organização obter sucesso no $m$-commerce. Esses fatores são discutidos nos trabalhos de Huang, Qi e Dong (2007), Sharma e Gutiérrez (2010), e Martín, López-Catalán e Ramón-Jerónimo (2012). Além dos já comentados, ainda há outros fatores citados na literatura (SHIH; HUANG, 2009).

Em resumo, como principais fatores críticos de sucesso no $m$-commerce pode-se dizer que estão a necessidade de um mercado disposto a utilizar o serviço, a inserção do $m$-commerce no planejamento estratégico da empresa, questões relacionadas à tecnologia disponível e utilizada e um modelo de negócios adequado. Assim, verifica-se que a oferta do serviço de m-commerce é uma tarefa que exige competência por parte de quem deseja efetivamente fazê-lo.

\section{CONSIDERAÇÕES FINAIS}

A discussão sobre m-commerce neste artigo buscou explorar um campo com espaço para desenvolvimento. A realização de uma diversidade de trabalhos focados no usuário final parece não fazer sentido sem a contraparte do foco nas empresas/organizações que oferecem o serviço. A discussão que a literatura traz pode ser considerada unilateral, pois ela discute o m-commerce muito mais numa perspectiva do usuário querer adotar, do que das empresas oferecerem. Por isso, foi objetivo desta pesquisa identificar vantagens e desafios que as organizações encontram ao oferecerem o serviço de $m$ commerce.

A pesquisa mostrou que a quantidade de trabalhos sobre $m$-commerce com o foco aqui buscado é pequena. Verificou-se que, dentre os trabalhos encontrados, não há foco em nenhum setor empresarial específico. Entretanto, mesmo que as pesquisas sejam direcionadas a diferentes setores, ou então teóricas, elas trazem resultados complementares entre si e proporcionam uma visão ampla sobre o $m$ commerce na perspectiva das empresas ofertantes do serviço.

Quanto aos resultados reportados pelos estudos analisados, cabem algumas considerações. Ao tratar dos fatores que levam uma organização a oferecer o serviço de $m$-commerce, constata-se que os motivos 
já são muito bem conhecidos na área da administração empresarial, ou seja, estão relacionados, basicamente, ao aumento de receitas e melhoria do relacionamento com os clientes. Certamente são questões plausíveis, já que têm contribuído para o sucesso de grande número de organizações com fins lucrativos.

A segunda questão, que trata dos benefícios e efeitos, é particular a determinados casos. A maior parte dos benefícios apontados tem contribuído positivamente para o sucesso de grandes organizações, que dispõe de recursos suficientes para criar uma estrutura que lhes permita efetivamente ter esses "benefícios". Pequenas organizações podem, sem dúvida, oferecer o serviço, mas poucas vezes têm condições de ter uma estrutura própria que transforme os dados dos clientes em informações úteis. Pelo menos no Brasil, essa é uma situação comum. Apenas para fins de exemplificação dessa diferença, propõe-se ao leitor comparar um aplicativo oferecido por uma empresa local, de pequeno porte, e o aplicativo da Amazon ${ }^{\circledR}$. Sem dúvida, este último apresenta um nível de personalização superior. Em resumo, os benefícios apontados nos trabalhos analisados não necessariamente são benefícios para qualquer organização, mas basicamente para as de grande porte. De certa forma, pequenas organizações podem até mesmo ser prejudicadas, a menos que estejam cientes de sua situação e saibam adotar alternativas, seja na oferta de um serviço diferenciado, ou por meio da associação com outras organizações, com interesses comuns.

Em relação ao terceiro ponto, que trata das barreiras que se impõe à oferta do serviço, verifica-se que algumas delas já foram amenizadas, mas, pelo menos no Brasil, ainda existem. Outras barreiras citadas não tendem a se amenizar, como é o caso da diversidade de sistemas operacionais e as diferenças nos padrões de tecnologia. Barreiras internas às organizações tendem a diminuir não com a evolução do m-commerce, mas com maior profissionalização da administração, como é o caso das barreiras relacionadas ao modelo de negócios.

Quanto aos fatores críticos de sucesso, observa-se que os resultados atingidos pelos trabalhos analisados remetem muito mais a questões de administração empresarial do que ao $m$ commerce propriamente dito. Muitos desses pontos são tratados em disciplinas da administração relacionadas ao planejamento empresarial e ao marketing. As questões de cunho tecnológico certamente já não constituem mais um dos maiores problemas, frente ao crescimento da capacidade computacional.

$\mathrm{O}$ que se apresentou neste trabalho são informações úteis para quem busca uma 
visão mais holística do mercado de $m$ commerce e não apenas uma "visão do usuário", como a maioria das pesquisas tem feito. O que foi apresentado pode ser tomado tanto como um guia para quem deseja oferecer o serviço de m-commerce, como um guia para pesquisas futuras que almejem analisar organizações ofertantes do serviço. A carência de estudos com esse foco ficou evidente. Assim, deixa-se como desafio para pesquisas futuras a identificação de setores e características empresariais que afetem, positivamente ou negativamente, a oferta do serviço de $m$ commerce, bem como barreiras e fatores críticos de sucesso.

\section{AGRADECIMENTO}

Os autores agradecem ao auxílio financeiro recebido da Fundação de Amparo à Pesquisa e Inovação do Estado de Santa Catarina (FAPESC) em forma de bolsa de estudos para pós-graduação stricto sensu (nível de mestrado).

\section{REFERÊNCIAS}

ALFAHL, H.; HOUGHTON, L.; SANZOGNI, L. Mobile Commerce adoption in organizations: a literature review and future research directions.

Journal of Electronic Commerce in Organizations, v. 10, p. 61+, 2012.

ALVES, M. T. V. D. Análise de conteúdo: sua aplicação nas publicações de contabilidade. Revista Universo Contábil, v. 7, n. 3, p. 146-166, 2011.
ANATEL. Acessos Telefonia Móvel: Móvel pessoal. 2016. Disponível em: $<$ http://ftp.anatel.gov.br/dados/Acessos/Mo vel_Pessoal/Total/csv/>. Acesso em: 18 nov. 2016.

BALASUBRAMANIAN, S.; PETERSON, R. A.; JARVENPAA, S. L. Exploring the implications of m-commerce for markets and marketing. Journal of the Academy of Marketing Science, v. 30, n. 4, p. 348361, 2002.

BARNETT, N.; HODGES, S.; WILSHIRE, M. M-commerce: an operator's manual. The McKinsey Quarterly, n. 3, p. 163-173, 2000.

BHEDA, N. M-commerce for the masses. Siliconindia, n. 40, July, 2010.

CHANG, M. K.; CHEUNG, W.; LAI, V. S. Literature derived reference models for the adoption of online shopping. Information \& Management, v. 42, p. 543-559, 2005.

CHONG, A. Y.-L. A two-staged SEMneural network approach for understanding and predicting the determinants of $\mathrm{m}$ commerce adoption. Expert Systems with Applications, v. 40, p. 1240-1247, 2013.

CHU, Y.; HUANG, L. Mobile

Technologies Adoption: An Exploratory Case Study. Tsinghua Science \&

Technology, v. 13, n. 3, p. 300-305, 2008.

CLARKE, I., III. Emerging value propositions for $\mathrm{m}$-commerce. Journal of Business Strategies, v. 25, n. 2, p. 41+, 2008.

E-BIT. Relatório WebShoppers. 31. ed., 2015. Disponível em: <http://www.webvenda.com/wpcontent/uploads/2015/02/31_webshoppers. pdf>. Acesso em: 18 nov. 2016. 
FROLICK, M. N.; CHEN, L. Assessing mcommerce opportunities. Information systems management. Spring, 2004.

GAN, L.; TU, W.; WANG, A. The Research on Application of Business Intelligence in Mobile E-commerce. Proceedings of the 2013 International Academic Workshop on Social Science (Iaw-Sc 2013), v. 50, p. 331-334, 2013.

GEORGIADIS, C. K. Mobile Commerce Application Development: Implementing Location-aware Information Services. In: GERACI, P.; LOGOTHETIS, M. Aict: 2009 Fifth Advanced International Conference on Telecommunications, $p$. 333-338, 2009.

GODOE, H.; HANSEN, T. B.

Technological regimes in $\mathrm{m}$-commerce: Convergence as a barrier to diffusion and entrepreneurship? Telecommunications Policy, v. 33, n. 1/2, p. 19-28, 2009.

HUANG, W.; QI, L. Y.; DONG, L. H. Business Models and Implementations of M-Commerce: Case Studies and Future Research Issues. 2007 International Conference on Wireless Communications, Networking and Mobile Computing, p. 3637-3640, 2007.

IVAN, I.; MILODIN, D.; ZAMFIROIU, A. Security of m-commerce transactions. Theoretical and Applied Economics, v. 20, n. 7, p. 59-76, 2013.

JIN, C. H.; VILLEGAS, J. Mobile phone user's behaviors: the motivation factors of the mobile phone user. International Journal of Mobile Marketing, v. 3, n. 2, p. 4-14, 2008.

JONKER, J.-W. M-commerce and mpayment: combining technologies.

Bedrijfswiskunde en informatica, p. 128, 2003.
JUKIC, N. et al. M-commerce: Analysis of impact on marketing orientation. In: KHOSROWPOUR, M. Issues and Trends of Information Technology Management in Contemporary Organizations, p. 305307, 2002.

KOUROUTHANASSIS, P. E.; GIAGLIS, G. M. Introduction to the special issue mobile commerce: the past, present, and future of mobile commerce research. International Journal of Electronic Commerce, v. 16, n. 4, p. 5-17, 2012.

LEE, C.-S.; HO, J. C. A Framework for Analyzing Business Model Innovation in Mobile Commerce. Journal of International Technology \& Information Management, v. 19, n. 4, p. 37-60, 2010.

LIN, J. et al. Understanding the evolution of consumer trust in mobile commerce: a longitudinal study. Information Technology \& Management, v. 15, n. 1, p. 37-49, 2014.

MALIK, A; KUMRA, R.; SRIVASTAVA, V. Determinants of consumer acceptance of m-commerce. South Asian Journal of Management, v. 20, n. 2, p. 102-126, 2013.

MARTÍN, S. S.; LÓPEZ-CATALÁN, B.; RAMÓN-JERÓNIMO, M. A. Factors determining firms' perceived performance of mobile commerce. Industrial Management \& Data Systems, v. 112, n. 6, p. 946-963, 2012.

MARTÍN-GUTIÉRREZ, S.; LÓPEZCATALÁN, S.; RAMÓN-JERÓNIMO, M. A. Determinants of involvement in mobile commerce: the moderating role of gender. EsicMarket, v. 141, p. 69-101, 2012.

MAY, P. Mobile Commerce:

Opportunities, Applications, and Technologies of Wireless Business. Breakthroughs in application development 
series. New York: Cambridge University Press, 2001. 304p.

NGAI, E.W.T.; GUNASEKARAN, A. Ecommerce in Hong Kong: an empirical perspective and analysis. Internet Research, v.15, n. 2, p. 141-159, 2005.

NGAI, E. W. T.; GUNASEKARAN, A. A review for mobile commerce research and applications. Decision Support Systems, v. 43, n. 1, p. 3-15, 2007.

PIGNEUR, Y. An ontology for m-business models. In: SPACCAPIETRA, S.; MARCH, S. T., et al. (Eds.). Conceptual Modeling - Er 2002, v. 2503, p. 3-6, 2002.

REICHOLD, A. et al. Case study - Mcommerce at Helsana Health Insurance: Mobile premium calculator. Proceedings of the 14th International Workshop on Database and Expert Systems Applications, p. 877-881, 2003.

SCHIERHOLZ, R.; KOLBE, L.; BRENNER, W. Strategy Alignment of Mobile Solutions in Customer-Oriented Processes. Wirtschaftsinformatik, v. 47, n. 1, 2005.

SHARMA, S.; GUTIÉRREZ, J. A. An evaluation framework for viable business models for $\mathrm{m}$-commerce in the information technology sector. Electronic Markets, v. 20, n. 1, p. 33-52, 2010.

SHIH, Y.-Y.; HUANG, S.-S. Exploring the critical success factors of mobile commerce via qualitative method- in case of insurance industry. Proceedings of the 2009 Sixth International Conference on Information Technology: New Generations, n. 1-3, p. 958-962, 2009.

TEZZA, R.; BORNIA, A. C.; ALBUQUERQUE, R. M. E-marketing e difusão no m-commerce. V Simpósio de Excelência em Gestão e Tecnologia
(SEGET). Resende - RJ: Associação Educacional Dom Bosco, 2008.

THAKUR, R.; SRIVASTAVA, M. Customer usage intention of mobile commerce in India: an empirical study. Journal of Indian Business Research, v. 5, n. 1, p. 52-72, 2013.

TIWARI, R.; BUSE, S. The mobile commerce prospects: a strategic analysis of opportunities in the banking sector. Hamburg: Hamburg University Press, 2007.

TOMÁŠKOVÁ, H. M-Commerce and Mbanking focused on Czech republic. In: MLADENOV, V. et al. (org.). Proceedings of the International Conference on Communication and Management in Technological Innovation and Academic Globalization, p.109-112, 2010.

VARNALI, K.; TOKER, A. Mobile marketing research: The-state-of-the-art. International Journal of Information Management, v. 30, n. 2, p. 144-151, 2010 .

VILLAS, M. V.; MACEDO-SOARES, T. D. L. A.; RUSSO, G. M. Bibliographical research method for business administration studies: a model based on scientific journal ranking. Brazilian Administration Review, v. 5, n. 2, p. 139159, 2008.

WU, J.-H.; WANG, S.-C. What drives mobile commerce? An empirical evaluation of the revised technology acceptance model. Information \& Management, v. 42, p. 719-729, 2005.

XU, H.; YANG, J. Do m-commerce user's expectations reflect reality? International Journal of Electronic Business Management, v. 10, n. 4, p. 322-331, 2012. 
YANG, K. C. C. Exploring factors

affecting the adoption of mobile commerce

in Singapore. Telematics and

Informatics, n. 22, p. 257-277, 2005.

YEO, J.; HUANG, W. Mobile Ecommerce Outlook. International Journal of Information Technology \& Decision Making, v. 2, n. 2, p. 313-332, 2003. 